\title{
Prospective randomised clinical trial to evaluate the safety and efficacy of nepafenac $0.1 \%$ treatment for the prevention of macular oedema associated with cataract surgery in patients with diabetic retinopathy
}

\author{
Ayala Pollack, ${ }^{1}$ Giovanni Staurenghi, ${ }^{2}$ Dana Sager, ${ }^{3}$ Bickol Mukesh, ${ }^{3}$ Harvey Reiser, ${ }^{4}$ \\ Rishi P Singh ${ }^{5}$
}

- Additional material is published online only. To view please visit the journal online (http://dx.doi.org/10.1136/ bjophthalmol-2016-308617).

'Department of Ophthalmology, Kaplan Medical Center, Rehovot Israel

${ }^{2}$ Department of Biomedical and Clinical Science, Luigi Sacco Hospital, University of Milan, Milan, Italy

${ }^{3}$ Alcon Research Ltd., Fort Worth, Texas, USA

${ }^{4}$ Eye Care Specialists, Kingston, Pennsylvania, USA

${ }^{5}$ Cole Eye Institute, Cleveland

Clinic, Cleveland, Ohio, USA

Correspondence to Dr Rishi P Singh, Cole Eye Institute, Cleveland Clinic, 9500 Euclid Avenue, i32 Cleveland, OH 44195, USA; drrishisingh@gmail.com

Received 26 February 2016 Revised 18 May 2016 Accepted 15 June 2016 Published Online First 7 July 2016

\section{(1) Corsinate}

To cite: Pollack $A$, Staurenghi G, Sager D, et al. Br J Ophthalmol 2017; 101:423-427.

\section{ABSTRACT}

Background/aims This study evaluated nepafenac ophthalmic suspension $0.1 \%$ for prevention of macular oedema (MO) when used 90 days following cataract surgery in patients with diabetic retinopathy (DR).

Methods Randomised, double-masked, vehiclecontrolled, parallel group study conducted at 32 centres across the world. Participants were patients with diabetes with non-proliferative diabetic retinopathy scheduled for cataract surgery with (posterior chamber) intraocular lens implantation. Patients were randomised to nepafenac ophthalmic suspension $0.1 \%$ or vehicle three times daily, beginning on the day before surgery and continuing through the last study visit (day 90 or early exit). All patients were instilled one drop of tobramycin $0.3 \%$ and dexamethasone $0.1 \%$ four times daily for 2 weeks after surgery. Primary efficacy end point was the percentage of patients who developed MO (defined as $\geq 30 \%$ increase in central subfield macular thickness from baseline) within 90 days following surgery. The secondary end point was mean change in best-corrected visual acuity (BCVA) from baseline to day 90.

Results A total of 175 patients were randomised, with 87 and 88 patients in the nepafenac and vehicle groups, respectively. A significantly greater percentage of eyes in the vehicle group (17.5\%; $95 \% \mathrm{Cl} 9.9 \%$ to $27.6 \%$ ) developed $\mathrm{MO}$ within 90 days following surgery compared with the nepafenac group $(5.0 \% ; 95 \% \mathrm{Cl}$ $1.4 \%$ to $12.3 \%, p=0.01$ ). Mean change in BCVA from baseline to day 90 following surgery was greater in the nepafenac group (17.7 \pm 14.6 letters) relative to the vehicle group (14.3 \pm 13.9 letters), though the difference was not statistically significant $(p=0.14)$. No new safety issues or trends were identified.

Conclusions A 90-day nepafenac treatment regimen prevented MO after cataract surgery in patients with $D R$ and demonstrated no safety issues within this study group.

Trial registration number NTC00782717 and NCT00939276.

\section{INTRODUCTION}

Macular oedema (MO) is a postoperative complication of cataract surgery that typically develops 4-12 weeks after the procedure. ${ }^{1}$ Approximately $2 \%$ of patients who undergo uncomplicated cataract surgery develop MO that is associated with vision loss. Anatomical assessments based on optical coherence tomography (OCT) suggest that MO develops in as many as $40 \%$ of eyes, though incidence rates vary depending on the presence of predisposing factors, such as diabetic retinopathy (DR) or uveitis, and the method of detection, whether OCT, fluorescein angiography or clinical examination. ${ }^{2-4}$ The incidence of MO associated with cataract surgery is higher among patients with diabetes compared with those without. More than $75 \%$ of patients with mild or moderate DR have vascular leakage after cataract surgery, and more than $3 \%$ of patients with diabetes develop MO with clinically significant vision loss within 12 months of surgery, regardless of DR status. ${ }^{4}{ }^{5}$ The threshold for developing MO may be lower in patients with diabetes due to hyperglycaemia that is associated with increased intraocular levels of advanced glycation end products, reactive oxygen species and proinflammatory factors that weaken retinal vasculature and disrupt the blood-retina barrier. $^{6}$

Inflammation associated with cataract surgery is routinely managed with a postoperative regimen of corticosteroids, non-steroidal anti-inflammatory drugs (NSAIDs) or a combination of both. NSAIDs reduce the activity of inflammatory pathways, including those that lead to MO, by inhibiting cyclo-oxygenase enzymes that convert cell membrane phospholipids to prostaglandin precursors. ${ }^{17}$ Clinical trials have been performed to compare the safety and efficacy of topical ophthalmic NSAIDs, including diclofenac, ketorolac, bromfenac and nepafenac, with corticosteroids or corticosteroid-NSAID combinations in the prevention of MO associated with cataract surgery. ${ }^{8-13}$ Overarching conclusions from these trials support a role for NSAIDs in prevention of $\mathrm{MO}$ associated with cataract surgery, but interpretation of the findings are limited as many of the studies did not include a vehicle control, included a small number of patients or followed postsurgical outcomes over a short duration (6 weeks or less).

Nepafenac ophthalmic suspension $0.1 \%$ (NEVANAC, Alcon Research, Fort Worth, Texas, USA) is a topical ophthalmic NSAID approved in multiple countries for the treatment of pain and inflammation associated with cataract surgery. ${ }^{14}$ Nepafenac is a prodrug that rapidly penetrates the 
cornea to achieve high ocular bioavailability in the aqueous humour. ${ }^{15}$ Intraocular hydrolases in the iris, ciliary body and retina convert nepafenac to amfenac, which is a potent inhibitor of cyclo-oxygenase activity. ${ }^{15} 16$ Amfenac has high ocular bioavailability in the aqueous humour, and in the vitreous. ${ }^{15} 17$ The safety and efficacy of an extended 90-day course of nepafenac $0.1 \%$ for the prevention of $\mathrm{MO}$ associated with cataract surgery in patients with DR is supported by the findings of a previously reported randomised, double-masked, vehiclecontrolled clinical trial. ${ }^{18}$

The primary objectives of this study were to evaluate the safety and efficacy of nepafenac $0.1 \%$ for the prevention of MO (defined as $\geq 30 \%$ increase in central subfield macular thickness (CSMT) from baseline ${ }^{18}{ }^{19}$ ) when used for 90 days following cataract surgery in patients with DR.

\section{MATERIALS AND METHODS}

\section{Study objective and methodology}

This was a multicentre, randomised, double-masked, vehiclecontrolled, parallel group study designed to evaluate the hypothesis that nepafenac $0.1 \%$ is safe and efficacious for the prevention of MO when used for 90 days following cataract surgery in patients with DR. Patients were evaluated on day 1 , day 7, day 14, day 30, day 60 and day 90 (or at time of early discontinuation from the study (ie, early exit)). Investigators determined whether patients should be discontinued from the study based on occurrence of the following criteria: CSMT increase of $30 \%$ or more from baseline, ${ }^{18}{ }^{19}$ best-corrected visual acuity (BCVA) decreases considered by the investigator to be associated with retinal thickening or presence of cystoid abnormalities detected by spectral domain OCT (SD-OCT).

The study was conducted in accordance with Good Clinical Practices and the ethical principles that have their origins in the Declaration of Helsinki. All participating patients provided their written informed consent. The study protocol and associated informed consent forms were reviewed and approved by institutional review boards and independent ethics committees for each investigational centre. More information on study methodology is in the online supplementary section.

\section{Efficacy assessments}

The primary outcome measure for this study was the percentage of patients who developed MO within 90 days following cataract surgery. The secondary outcome measure was mean change in BCVA from baseline to day 90. Macular thickness and macular volume were measured for the study eye using SPECTRALIS SD-OCT (Heidelberg Engineering, Carlsbad, California, USA) or Zeiss Cirrus SD-OCT (Carl Zeiss Meditec, Dublin, California, USA) at the screening visit and on day 7, day 14, day 30, day 60 and day 90 (or early exit). BCVA was assessed at each visit using the standardised Early Treatment Diabetic Retinopathy Study (ETDRS) procedure requiring certified refractionists, examiners and rooms. Study eye assessments were conducted at the baseline visit and on day 1 , day 7, day 14, day 30, day 60 and day 90 (or early exit). Manifest refraction was preferred on day 1 but pinhole correction may have been performed at the discretion of the investigator with documentation.

\section{Safety assessments}

Information on adverse events (AEs) was collected for all patients after the first administration of study drug on day 1 and during all postoperative visits through day 90 (or early exit).
More information on the safety assessment is in the online supplementary section.

\section{Study population}

This study was planned to enrol approximately 222 evaluable patients (111 patients in each treatment group) to have $90 \%$ power to detect a 50\% reduction in the incidence of $\mathrm{MO}$ (42\% to $21 \%$ ) within 90 days of cataract surgery for nepafenac $0.1 \%$ relative to vehicle. The sample size for this study was guided by a MO incidence rate of $42 \%$ in patients with DR. ${ }^{20}$

Between August 2009 and August 2011, study investigators enrolled and treated 175 patients (first patient screened to last patient exited) at 32 investigational centres across Europe, India, Israel, New Zealand and the USA. As a result of patient recruitment difficulties, enrolment was terminated in May 2011. Patients who had been randomised and were undergoing treatment were allowed, at the discretion of the investigator, to complete the study as planned but no new patients were screened or enrolled after this point. Of the 175 patients, 9 patients did not undergo surgery and were excluded from both the safety and intent-to-treat (ITT) population and another 6 patients that did not have any postbaseline visit were excluded from the ITT population. A total of 160 patients were included in the ITT analysis population and 166 patients were included in the safety analysis population (see online supplementary figure S1). More information on the patient population is in the online supplementary section.

Table 1 Patient demographics and ocular characteristics at baseline, by treatment group

\begin{tabular}{|c|c|c|c|}
\hline & $\begin{array}{l}\text { Nepafenac } \\
(\mathrm{N}=80)\end{array}$ & $\begin{array}{l}\text { Vehicle } \\
(\mathrm{N}=80)\end{array}$ & $\begin{array}{l}p \\
\text { Value }\end{array}$ \\
\hline \multicolumn{4}{|l|}{ Age (years) } \\
\hline Mean (SD) & $68.1(8.6)$ & $69.4(7.6)$ & \multirow[t]{2}{*}{0.31} \\
\hline Min, Max & 49,90 & 53,84 & \\
\hline \multicolumn{4}{|l|}{ Sex, n (\%) } \\
\hline Male & $51(63.8)$ & $44(55.0)$ & \multirow[t]{2}{*}{0.26} \\
\hline Female & $29(36.3)$ & $36(45.0)$ & \\
\hline \multicolumn{4}{|l|}{ Race, n (\%) } \\
\hline Asian & $6(7.5)$ & $5(6.3)$ & \multirow[t]{6}{*}{0.97} \\
\hline Black or African-American & $1(1.3)$ & $0(0.0)$ & \\
\hline Native Hawaiian or Other & $2(2.5)$ & $2(2.5)$ & \\
\hline Pacific Islander & $0(0.0)$ & $0(0.0)$ & \\
\hline Other & $2(2.5)$ & $2(2.5)$ & \\
\hline White & $69(86.3)$ & $71(88.8)$ & \\
\hline \multicolumn{4}{|l|}{ NPDR classification, $\mathrm{n}(\%)$} \\
\hline Mild NPDR & $58(72.5)$ & $57(71.3)$ & \multirow[t]{3}{*}{1.0} \\
\hline Moderate NPDR & $21(26.3)$ & $22(27.5)$ & \\
\hline Severe NPDR & $1(1.3)$ & $1(1.3)$ & \\
\hline \multicolumn{4}{|l|}{ BCVA* (ETDRS letters) } \\
\hline Mean (SD) & $63.3(12.8)$ & $65.2(12.1)$ & \multirow[t]{2}{*}{0.34} \\
\hline Min, Max & 13,84 & 28,84 & \\
\hline \multicolumn{4}{|l|}{$\operatorname{CSMT}(\mu \mathrm{m})$} \\
\hline Mean (SD) & $268.8(29.0)$ & $276.8(23.2)$ & \multirow[t]{2}{*}{0.06} \\
\hline Min, Max & 182,371 & 224,364 & \\
\hline \multicolumn{4}{|c|}{$\begin{array}{l}\text { *Baseline BCVA values based on } 79 \text { patients in the nepafenac group and } 79 \text { patients } \\
\text { in the vehicle group. } \\
\text { Nepafenac=nepafenac ophthalmic suspension, } 0.1 \% \text {; Vehicle=nepafenac ophthalmic } \\
\text { suspension, vehicle. } \\
\text { BCVA, best-corrected visual acuity; CSMT, central subfield macular thickness; ETDRS, } \\
\text { Early Treatment Diabetic Retinopathy Study; NPDR, non-proliferative diabetic } \\
\text { retinopathy. }\end{array}$} \\
\hline
\end{tabular}




\section{Statistical analysis}

Details on the statistical analysis can be found in the online supplementary section.

\section{RESULTS}

\section{Patient disposition and characteristics}

Demographic and baseline characteristics were generally similar between treatment groups for patients in the ITT analysis population (table 1). Mean patient age was $68.1 \pm 8.6$ years in the nepafenac group and $69.4 \pm 7.6$ years in the vehicle group. Most patients had non-proliferative diabetic retinopathy (NPDR) that was classified as mild $(72.5 \%$ in the nepafenac group and $71.3 \%$ in the vehicle group). Mean baseline CSMT was 268.8 $\pm 29.0 \mu \mathrm{m}$ in the nepafenac group and $276.8 \pm 23.2 \mu \mathrm{m}$ in the vehicle group. Mean baseline BCVA was $63.3 \pm 12.8$ letters in the nepafenac group and $65.2 \pm 12.1$ letters in the vehicle group.

\section{MO and central subfield macular thickness}

A significantly greater percentage of patients developed MO within 90 days following cataract surgery in the vehicle group $(\mathrm{n}=14 / 80,17.5 \%$; $95 \%$ CI $9.9 \%$ to $27.6 \%)$ compared with the nepafenac group $(\mathrm{n}=4 / 80,5.0 \% ; 95 \%$ CI $1.4 \%$ to $12.3 \%$, $\mathrm{p}=0.01$ ). Mean CSMT was increased from baseline at most postsurgical visits in both treatment groups, but increases were markedly lower in the nepafenac group compared with the vehicle group starting at day 30 following cataract surgery (figure 1). CSMT returned to $\pm 10 \%$ of baseline within 60 days following cataract surgery in $91.0 \%(n=61 / 67)$ of patients in the nepafenac group compared with $55.6 \%(n=35 / 63)$ of patients in the vehicle group.

\section{Postsurgical visual outcomes}

Mean BCVA change from preoperative baseline to day 90 following cataract surgery (key secondary end point) was greater in the nepafenac group (17.7 \pm 14.6 letters) relative to the vehicle group (14.3 \pm 13.9 letters), though the difference was not statistically significant $(p=0.14)$.

Mean BCVA change from preoperative baseline to day 90 following cataract surgery was greater in the nepafenac group compared with the vehicle group regardless of whether patients had developed MO at any visit after surgery, though the differences were not statistically significant (table 2).

Among patients who had $\mathrm{MO}$ at any visit after surgery, mean BCVA change from preoperative baseline to day 90 following cataract surgery was $16.3 \pm 22.1$ letters in the nepafenac group and $8.4 \pm 15.4$ letters in the vehicle group $(p=0.34)$. Among patients who did not have MO at any visit after surgery, mean BCVA change from preoperative baseline to day 90 following cataract surgery was $17.8 \pm 14.3$ letters in the nepafenac group and $15.3 \pm 13.5$ letters in the vehicle group $(p=0.29)$.

From day 7 to day 90 following cataract surgery, BCVA decreased $>5$ letters in $13.8 \%(n=11 / 80)$ of patients in the vehicle group and $6.3 \%(\mathrm{n}=5 / 79)$ of patients in the nepafenac group. Among patients who developed $\mathrm{MO}$ after cataract surgery, BCVA decreased $>10$ letters from day 7 to any following study visit in $50.0 \%(\mathrm{n}=7 / 14)$ of patients in the vehicle group and $0.0 \%(\mathrm{n}=0 / 4)$ of patients in the nepafenac group.

\section{Safety}

The incidences of special-interest AEs were generally similar between treatment groups (table 3). Seven cases of punctate
Figure 1 Central subfield macular thickness changes from baseline at each study visit (intent-to-treat population). Mean central subfield thickness was increased from baseline at most postsurgical visits in both treatment groups, but increases were markedly lower in the nepafenac group compared with the vehicle group starting at day 30 following cataract surgery. Sample sizes for the nepafenac group were 73 (day 7), 78 (day 14), 79 (day 30) and 80 (day 60 and day 90). Sample sizes for the vehicle group were 76 (day 7), 79 (day 14) and 80 (day 30 , day 60 , day 90 ). $\mathrm{BL}$, baseline.

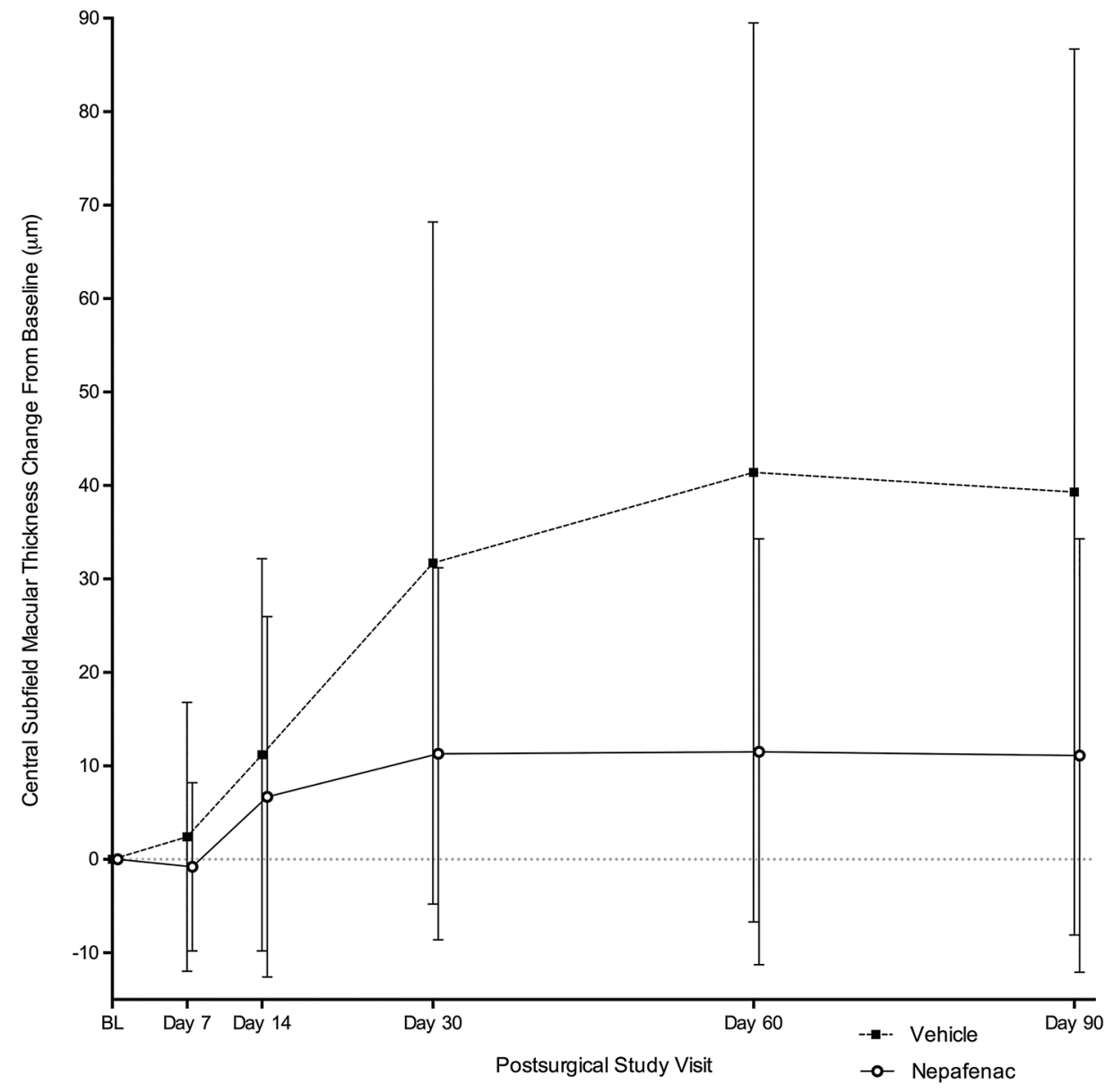


Table 2 BCVA changes from preoperative baseline to day 90 after cataract surgery, by treatment group and macular oedema status (intent-to-treat analysis population)

\begin{tabular}{|c|c|c|c|c|}
\hline Treatment group & Total & $\begin{array}{l}\text { Mean BCVA } \\
\text { change (SD) }\end{array}$ & $95 \% \mathrm{Cl}$ & p Value \\
\hline \multicolumn{5}{|l|}{ MO } \\
\hline Nepafenac & 4 & $16.3(22.1)$ & -18.8 to 51.3 & 0.34 \\
\hline Vehicle & 11 & $8.4(15.4)$ & -2.0 to 18.7 & \\
\hline \multicolumn{5}{|l|}{ No MO } \\
\hline Nepafenac & 75 & $17.8(14.3)$ & 14.5 to 21.1 & 0.29 \\
\hline Vehicle & 68 & $15.3(13.5)$ & 12.0 to 18.5 & \\
\hline
\end{tabular}

keratitis (four and three in the nepafenac and vehicle groups, respectively) and one case of keratitis (vehicle) were reported. Three corneal events were reported (one case each of corneal oedema and disorder in the vehicle group and one of corneal erosion in the vehicle group). Two of the non-serious AEs that led to discontinuation in the nepafenac group were considered to be treatment-related: corneal erosion and allergic dermatitis.

No new safety issues or trends were identified based on review of the incidence, seriousness, intensity, onset, duration, outcome, relationship to treatment or discontinuation due to AEs, or assessments of changes from baseline in corneal staining, intraocular pressure, ocular signs or fundus parameters.

\section{DISCUSSION}

This multicentre, randomised, double-masked, vehiclecontrolled, parallel-group study validated the hypothesis that nepafenac $0.1 \%$ is safe and efficacious for the prevention of MO when used for 90 days following cataract surgery in

Table 3 Summary of treatment-emergent adverse events (safety population)

\begin{tabular}{|c|c|c|c|c|}
\hline \multirow[b]{2}{*}{ Adverse event category } & \multicolumn{2}{|c|}{$\begin{array}{l}\text { Nepafenac } \\
(\mathrm{N}=83)\end{array}$} & \multicolumn{2}{|c|}{$\begin{array}{l}\text { Vehicle } \\
(\mathrm{N}=83)\end{array}$} \\
\hline & $\mathbf{N}$ & $\begin{array}{l}\text { Per } \\
\text { cent }\end{array}$ & $\mathbf{N}$ & $\begin{array}{l}\text { Per } \\
\text { cent }\end{array}$ \\
\hline Patients discontinued due to an $\mathrm{AE}$ & 4 & 4.8 & 7 & 8.4 \\
\hline Discontinued due to a non-fatal SAE & 1 & 1.2 & 2 & 2.4 \\
\hline Discontinued due to a non-serious $\mathrm{AE}$ & 3 & 3.6 & 5 & 6.0 \\
\hline Treatment-related & 2 & 2.4 & 2 & 2.4 \\
\hline Not related to treatment & 1 & 1.2 & 3 & 3.6 \\
\hline $\begin{array}{l}\text { Patients with at least one treatment-emergent } A E \\
\text { related to treatment (ADR) }\end{array}$ & 6 & 7.2 & 6 & 7.2 \\
\hline \multicolumn{5}{|l|}{ Most frequent ADRs (incidence of $1 \%$ or greater) } \\
\hline Corneal oedema & 0 & 0.0 & 1 & 1.2 \\
\hline Punctate keratitis & 4 & 4.8 & 3 & 3.6 \\
\hline Corneal disorder & 0 & 0.0 & 1 & 1.2 \\
\hline Corneal erosion & 1 & 1.2 & 0 & 0.0 \\
\hline Keratitis & 0 & 0.0 & 1 & 1.2 \\
\hline Conjunctival hyperaemia & 0 & 0.0 & 1 & 1.2 \\
\hline Eye irritation & 0 & 0.0 & 1 & 1.2 \\
\hline Dermatitis allergic & 1 & 1.2 & 0 & 0.0 \\
\hline
\end{tabular}

patients with DR. A significantly greater percentage of patients in the vehicle group developed MO within 90 days of cataract surgery compared with patients in the nepafenac group.

$\mathrm{MO}$ was defined as an increase of $30 \%$ or more in CSMT relative to the preoperative baseline measurement. This threshold has been determined to be appropriate for identifying clinically relevant changes in patients and is well above the 10\% coefficient of variation associated with OCT repeat testing variability. ${ }^{18} 1921$ The use of CSMT to determine MO was based on findings from a previous study indicating this measure is more reliable than centre-point macular thickness. ${ }^{20}$ The beneficial effect of nepafenac in maintaining macular thickness after cataract surgery is also supported by the finding that CSMT was near baseline levels by day 60 following cataract surgery in more than $90 \%$ of patients in the nepafenac group compared with only slightly more than half of the patients in the vehicle group. No trends were observed that indicated greater or lesser efficacy among demographic or NPDR severity subgroups, though conclusions cannot be drawn from this analysis due to the small numbers of patients who developed MO in this study.

Visual outcomes were numerically better in the nepafenac group compared with the vehicle group, though none of the observed differences were statistically significant. This lack of statistical significance was likely due to only $67 \%$ of the planned total patient population being enrolled after early termination of recruitment (study enrolment was terminated early due to difficulties in patient recruitment). Despite the lack of significance, the results reveal a trend that favours nepafenac compared with vehicle in BCVA change from baseline to day 90 following cataract surgery.

The results of this study are supported by the findings of a previously reported randomised, double-masked, vehiclecontrolled clinical trial designed to test the hypothesis that extended treatment with nepafenac $0.1 \%$ is safe and efficacious for the prevention of $\mathrm{MO}$ following cataract surgery in patients with DR. ${ }^{18}$ This is especially notable considering that retinal anatomy was assessed with different types of OCT in each study: SD-OCT in the current study versus time domain OCT (TD-OCT) in the former. A higher percentage of screened patients were excluded from the current study despite the use of similar enrolment criteria across each trial, likely because the greater sensitivity of SD-OCT versus TD-OCT supports an increased ability to detect intraretinal anatomical features, such as cystoid abnormalities, that were cause for exclusion. Inclusion of these patients, with more severe retinopathy, may have allowed for enrolment to be completed and possibly shown a greater difference from vehicle in the end points.

Application of topical ophthalmic nepafenac is a non-invasive approach for preventing $\mathrm{MO}$ associated with cataract surgery in patients with diabetes. This is in contrast to the range of currently used off-label treatments for the prevention of MO following surgery, which are all administered by intravitreal injection. These include anti-vascular endothelial growth factor (VEGF) options, such as ranibizumab (LUCENTIS, Genentech, South San Francisco, California, USA), bevacizumab (Avastin, Genentech, South San Francisco, California, USA) and aflibercept, and sustained-release corticosteroid implants that release dexamethasone (OZURDEX, Allergan, Irvine, California, USA). ${ }^{22}$

Nepafenac was generally well tolerated in the safety population. Treatment-related AEs in the nepafenac group included four cases of punctate keratitis and one case of allergic dermatitis. Corneal erosion has been labelled as an NSAID class effect, and was therefore targeted as an $\mathrm{AE}$ of special interest in the study. As such, exclusion criteria were added to prevent 
enrolment of patients with compromised corneas. ${ }^{23} 24$ One case of corneal erosion in the nepafenac group was assessed by the investigator as related to study treatment (out of two reported). The patient was discontinued from the study due to this event and the event resolved with treatment within 2 weeks after onset. Nepafenac is currently approved in multiple countries to treat pain and inflammation associated with cataract surgery for the first 2 weeks of the postoperative period. It is also approved in the European Union for the reduction in risk of postoperative MO associated with cataract surgery in patients with diabetes with treatment duration of 60 days. This study confirms that no new safety issues or trends are associated with an extended 90-day course of nepafenac following cataract surgery.

Findings from this study support the benefit of extended treatment with topical ophthalmic nepafenac for the prevention of MO associated with cataract surgery in patients with DR.

Acknowledgements The authors thank Alcon Research Ltd for the writing support provided and Meridius Health Communications that executed it.

Contributors AP and DS contributed to conception and design, data acquisition, data analysis, interpretation of data, critical revision and final approval. GS contributed to data acquisition, critical revision and final approval. BM contributed to conception and design, analysis and interpretation of data, drafting manuscript, critical revision and final approval. HR contributed to data acquisition and final approval. RPS contributed to conception and design, interpretation of data, drafting manuscript, critical revision and final approval.

Funding This study was sponsored and funded by Alcon Research.

Competing interests GS reports personal fees from Alcon, during the conduct of the study. DS reports others from Alcon Research Ltd, outside the submitted work. HR reports personal fees from Alcon, during the conduct of the study. RPS reports grants and personal fees from Alcon, grants and personal fees from Genentech, grants and personal fees from Regeneron, personal fees from Shire, during the conduct of the study.

Ethics approval Institutional Review Board for each investigational centre.

Provenance and peer review Not commissioned; externally peer reviewed.

\section{REFERENCES}

1 Lobo C. Pseudophakic cystoid macular edema. Ophthalmologica 2012;227:61-7.

2 Henderson BA, Kim JY, Ament CS, et al. Clinical pseudophakic cystoid macular edema. Risk factors for development and duration after treatment. J Cataract Refract Surg 2007:33:1550-8.

3 Lobo CL, Faria PM, Soares MA, et al. Macular alterations after small-incision cataract surgery. J Cataract Refract Surg 2004;30:752-60.

4 Schmier JK, Halpern MT, Covert DW, et al. Evaluation of costs for cystoid macular edema among patients after cataract surgery. Retina 2007;27:621-8.

5 Eriksson U, Alm A, Bjärnhall G, et al. Macular edema and visual outcome following cataract surgery in patients with diabetic retinopathy and controls. Graefes Arch Clin Exp Ophthalmol 2011;249:349-59.
6 Shin ES, Sorenson CM, Sheibani N. Diabetes and retinal vascular dysfunction. J Ophthalmic Vis Res 2014:9:362-73.

7 Kim SJ, Flach AJ, Jampol LM. Nonsteroidal anti-inflammatory drugs in ophthalmology. Surv Ophthalmol 2010:55:108-33.

8 Almeida DR, Johnson D, Hollands $\mathrm{H}$, et al. Effect of prophylactic nonsteroidal antiinflammatory drugs on cystoid macular edema assessed using optical coherence tomography quantification of total macular volume after cataract surgery. J Cataract Refract Surg 2008;34:64-9.

9 Asano S, Miyake K, Ota I, et al. Reducing angiographic cystoid macular edema and blood-aqueous barrier disruption after small-incision phacoemulsification and foldable intraocular lens implantation: multicenter prospective randomized comparison of topical diclofenac $0.1 \%$ and betamethasone $0.1 \%$. J Cataract Refract Surg 2008;34:57-63.

10 Endo N, Kato S, Haruyama K, et al. Efficacy of bromfenac sodium ophthalmic solution in preventing cystoid macular oedema after cataract surgery in patients with diabetes. Acta Ophthalmol 2010;88:896-900.

11 Miyake K, Nishimura K, Harino S, et al. The effect of topical diclofenac on choroidal blood flow in early postoperative pseudophakias with regard to cystoid macular edema formation. Invest Ophthalmol Vis Sci 2007:48:5647-52.

12 Miyake K, Ota I, Miyake G, et al. Nepafenac $0.1 \%$ versus fluorometholone $0.1 \%$ for preventing cystoid macular edema after cataract surgery. J Cataract Refract Surg 2011;37:1581-8.

13 Wittpenn JR, Silverstein S, Heier J, et al. A randomized, masked comparison of topical ketorolac $0.4 \%$ plus steroid vs steroid alone in low-risk cataract surgery patients. Am J Ophthalmol 2008; 146:554-60.

14 NEVANAC [package insert]. Fort Worth, TX: Alcon Laboratories, Inc., 2011.

15 Walters $T$, Raizman $M$, Ernest $P$, et al. In vivo pharmacokinetics and in vitro pharmacodynamics of nepafenac, amfenac, ketorolac, and bromfenac. J Catarac Refract Surg 2007;33:1539-45.

16 Ke TL, Graff G, Spellman JM, et al. Nepafenac, a unique nonsteroidal prodrug with potential utility in the treatment of trauma-induced ocular inflammation: II. In vitro bioactivation and permeation of external ocular barriers. Inflammation $2000: 24: 371-84$

17 Heier JS, Awh CC, Busbee BG, et al. Vitreous nonsteroidal antiinflammatory drug concentrations and prostaglandin E2 levels in vitrectomy patients treated with ketorolac $0.4 \%$, bromfenac $0.09 \%$, and nepafenac $0.1 \%$. Retina 2009:29:1310-13.

18 Singh R, Alpern L, Jaffe GJ, et al. Evaluation of nepafenac in prevention of macular edema following cataract surgery in patients with diabetic retinopathy. Clin Ophthalmol 2012;6:1259-69.

19 Kim SJ, Equi R, Bressler NM. Analysis of macular edema after cataract surgery in patients with diabetes using optical coherence tomography. Ophthalmology 2007;114:881-9.

20 Krzystolik MG, Strauber SF, Aiello LP, Diabetic Retinopathy Clinical Research Network, et al. Reproducibility of macular thickness and volume using Zeiss optical coherence tomography in patients with diabetic macular edema. Ophthalmology 2007; 114:1520-5.

21 Kim SJ, Belair ML, Bressler NM, et al. A method of reporting macular edema after cataract surgery using optical coherence tomography. Retina 2008;28:870-6.

22 Das A, McGuire PG, Rangasamy S. Diabetic macular edema: pathophysiology and novel therapeutic targets. Ophthalmology 2015;122:1375-94.

23 Gaynes BI, Fiscella R. Topical nonsteroidal anti-inflammatory drugs for ophthalmic use: a safety review. Drug Saf 2002;25:233-50.

24 Gaynes BI, Onyekwuluje A. Topical ophthalmic NSAIDs: a discussion with focus on nepafenac ophthalmic suspension. Clin Ophthalmol 2008:2:355-68. 